\title{
REFEXIONES SOBRE LA CONSTRUCCIÓN DE LA PERCEPCIÓN DE EXCLUSIÓN SOCIAL EN JÓVENES DE ENSEÑANZA MEDIA: PRECISIONES CONCEPTUALES Y METODOLÓGICAS
}

\author{
XIMENA ROJAS RETAMAL
}

\begin{abstract}
RESUMEN
El presente artículo da cuenta de los resultados de una indagación exploratoria, de carácter cualitativa, respecto de la construcción de la percepción de inclusión y/o exclusión social en jóvenes escolares y de edad escolar. Para ello, se realizaron entrevistas a diversos especialistas de las ciencias sociales. Dicho trabajo, permitió obtener precisiones respecto de lo relacional, contextual y temporal del concepto de inclusión y/o exclusión social. Así mismo, dio cuenta de la necesidad de mirar la escuela no como un espacio en sí mismo excluye, ya que son la mayoría de los jóvenes los que participan de la escuela, sino habría que mirar dicho espacio como parte de un sistema más amplio, un sistema social que excluye o incluye, un sistema social que fomenta expectativas respecto de los trayectos de vida, pero que no necesariamente genera las oportunidades para alcanzar dichas expectativas.
\end{abstract}

PALABRAS CLAVE: INCLUSIÓN SOCIAL, EXCLUSIÓN SOCIAL, JÓVENES ESCOLARES

* Académica del Departamento de Psicología, Universidad Católica Silva Henríquez, Santiago, Chile. E-Mail: xrojas@ucsh.cl. 


\begin{abstract}
RESUMO
O presente artigo apresenta os resultados de uma pesquisa exploratória qualitativa a respeito da construção da percepção de inclusão e/ou exclusão social em jovens estudantes e jovens em idade escolar. Para isto, foram realizadas entrevistas com diversos especialistas das ciências sociais. Foi possível obter precisões a respeito dos aspectos relacional, contextual e temporal do conceito de inclusão e/ou exclusão social. Da mesma forma, o estudo mostrou a necessidade de um outro olhar para a escola, não como um espaço que em si mesmo exclui, considerando que a maioria de seus participantes são os próprios jovens, mas como parte de um sistema mais amplo, um sistema social que exclui ou inclui, um sistema social que fomenta expectativas sobre os trajetos de vida, mas que não necessariamente gera as oportunidades para que tais expectativas sejam alcançadas.
\end{abstract}

PALAVRAS CHAVE: INCLUSÃO SOCIAL, EXCLUSÃO SOCIAL, JOVENS ESCOLARES

\begin{abstract}
This article takes into account the results of an exploratory study that is qualitative in nature, regarding perceptions of social inclusion and/or exclusion among students and school-age children. In carrying out the study, interviews were conducted with various experts in the social sciences. This project allowed us to obtain precise data on relational, contextual and temporal aspects of the concept of social inclusion and/or exclusion. In addition, it indicated the need for examining schools, not as a place of exclusion per se, since most young people take part in school. Instead school should be considered as part of a larger system, a social system, that excludes or includes people; a social system that fosters expectations regarding the life paths to be taken, but that does not necessarily generate opportunities for fulfilling these expectations.
\end{abstract}

KEY WORDS: SOCIAL INCLUSION, SOCIAL EXCLUSION, YOUNG SCHOLARS 


\section{INTRODUCCIÓN}

EL TRABAJO QUE SE desarrolla a continuación, corresponde a una reflexión respecto de algunos aspectos que se consideran centrales para el abordaje de la temática exclusión social en jóvenes. Dicha reflexión se enmarca en un proyecto de investigación que busca comprender desde la experiencia escolar de jóvenes de enseñanza media, la construcción de la percepción de exclusión social. ${ }^{1}$ Cabe señalar, que a raíz de las reflexiones y análisis realizado a partir del discurso de especialistas acerca del tema, fueron adecuados o modificados ciertos aspectos, los que incluyen, dentro de otros que serán mencionados en la medida que se desarrolla el presente artículo, incluye la modificación del título de la investigación original.

En el mundo de hoy, los escenarios sociales tienen el sello de la globalización, el individualismo, lo inmediato, la diversidad, lo incierto, todas características de lo que se ha denominado época de la modernidad o post modernidad para otros. En este contexto, la educación sigue representando una fuente inagotable y central en la integración social. Desde aquí, la preocupación por incorporar explícitamente políticas públicas que hablen de la equidad, la igualdad de oportunidades, la inclusión escolar, la formación en valores, la formación del ciudadano, de la convivencia social o el respeto por los que te rodean y por la naturaleza.

Cuando se habla de exclusión social, varios son los conceptos que aparecen asociados a éste, entre otros: tales como inclusión, discriminación, desigualdad e igualdad de oportunidades. Dicho fenómeno y sus asociaciones o precisiones conceptúales, han sido centro de preocupación en forma especial para la educación en Chile, si bien es una temática que implica todo el quehacer social, para la educación cobra especial vigencia.

1 El objetivo de dicho proyecto, forma parte del proyecto general «Construcción del sentimiento de exclusión social en la escuela: un análisis desde la experiencia escolar juvenil» a ser presentado en el concurso Fondecyt de Iniciación del año 2008. El fin de este trabajo, es poder adelantar en la reflexión y la búsqueda de información relevante para el proyecto general. De esta manera, presentar trabajo adelantado, que permita, finalizadas estas tareas, proyectar aún más el proyecto general y reformularlo en función de plantear posibles comparaciones según estratos socioeconómicos u otras dependencias de establecimiento. 
Sin duda, Chile ha realizado esfuerzos y cambios importantes dentro de su sistema educativo, de forma de responder tanto al mundo y al mercado globalizado y competitivo, como a las exigencias que esto implica en cuanto a lo ético y a la imagen que proyecta el comportamiento de la sociedad y de sus instituciones. De esta forma señala Molina:

Una sociedad en que predomina la justicia y la verdad, la responsabilidad personal en el trabajo bien hecho, la honestidad en los procedimientos y en el cumplimiento de los compromisos, la solución de los conflictos por medio del diálogo y no la confrontación, la capacidad de desarrollar el trabajo en equipo, el respeto de los derechos humanos, el funcionamiento de la democracia y de sus instituciones, en fin aquellos valores que hacen creíble, respetable y dan un sello a la identidad nacional, constituyen una base sólida para lograr el verdadero desarrollo de un país (Molina, 2003:326).

Específicamente, en el discurso de inauguración del año preescolar del 2003, el Ministro de Educación de la época declara:

La riqueza de Chile es la educación y nuestra meta va a ser poner a la educación en el centro del debate nacional. El arma más potente para combatir la desigualdad es la educación, el arma más potente para desarrollar a Chile es la educación. Si ustedes — niños, niñas y jóvenestienen mejor educación, lograría mejores empleos y tendrían mejores sueldos.

En este plano, aparecen datos, posturas, planteamientos e investigaciones que ponen en tensión el rol de la educación y específicamente las acciones intencionadas desde las políticas públicas en función de alcanzar las metas propuestas. Desde investigaciones como las de Rojas (2006), Assael y Cerda (1998) entre otras, existen ciertas evidencias que señalan desde el discurso de jóvenes escolares, que la institución escolar no representa necesariamente un espacio socializador positivo, señalan que la institución escolar no los legitima como sujetos con opinión, con intereses; de alguna manera desde su experiencia escolar ésta los excluye o discrimina, en definitiva, el escenario se tensiona, mientras la política declara intenciones y metas, los jóvenes declaran desconformismo y falta de sentido.

La investigación original, teniendo como marco que desde la política pública pareciera ser el educativo un escenario propicio para 
tratar el tema, busca profundizar en lo anteriormente mencionado, esto es, avanzar en dar respuesta a la pregunta ¿de qué manera son impactados los jóvenes en la institución escolar en la construcción del sentimiento de exclusión social? Busca comprender al sujeto juvenil escolar por un lado, y por otro, comparar estos discursos entre estudiantes de escuelas con población homogénea vulnerable de aquellos establecimientos que incorporan la cuota obligatoria de estudiantes de sectores definidos como de riesgo social, vulnerables, ${ }^{2}$ es decir, busca comparar discursos juveniles venidos de contextos escolares con integración u homogéneos.

Originalmente, ésta es una propuesta que se instala desde un enfoque cualitativo de investigación, dado que su objetivo se relaciona con los sentidos, el significado que le dan los jóvenes de enseñanza media a su experiencia escolar y cómo dentro de ésta se construyen sentimientos y percepciones de exclusión social, es decir, comprender cuales son aquellos ámbitos de la escuela que el joven escolar significa como constituyendo, aportando a la generación del sentimiento de exclusión social. De alguna manera, esta investigación aportaría a la (iluminación de los énfasis) caracterización de los posibles variables en juego en la construcción de tal sentimiento. Por tanto, que elementos debiesen estar a la base de la reflexión sobre políticas públicas, y respecto de cómo están impactando las intervenciones ministeriales. Se busca entonces constituir un aporte a la reflexión sobre la calidad y equidad de la educación, dos grandes conceptos que han estado a la base la Reforma Educacional y sus actualizaciones.

Avanzar en una propuesta investigativa, implica afinar y precisar aspectos tanto teóricos como metodológicos de la misma. Para lo anterior, se buscó a distintos especialistas ${ }^{3}$ de las ciencias sociales que

2 Proyecto de ley que introduce modificaciones a la ley 19.532 .

3 Por el carácter de esta primera parte del estudio, se han entrevistado más especialistas de lo previsto, a propósito de poder levantar tensiones o temas posibles de tensión en relación al discurso juvenil, así como también tener un acercamiento desde los diferentes especialistas a conceptos clave como el de exclusión propiamente tal, equidad, calidad educativa y discriminación. Se han entrevistado a los siguientes especialistas:

Jorge Baeza, sociólogo, doctor en ciencias de la educación, académico UCSH.

Oscar Dávila, trabajador social, doctorando en estudio de las sociedades latinoamericanas, investigador CIDPA. 
desde su experiencia académica, investigativa o de terreno, estuvieran cercanos y familiarizados ya sea con el mundo juvenil, el mundo educativo y escolar o ambos.

Para darle un orden comprensivo al desarrollo de las tensiones que se levantan a partir del objeto de estudio, se desarrollará en primer lugar, los aspectos teóricos puestos en discusión para dar paso a los aspectos metodológicos que decantan del desarrollo conceptual analítico anterior. A continuación se presentará lo que los especialistas fueron señalando en su discurso. Cada subtítulo, da cuenta de algunas de las categorías que se levantaron a partir de éste, así como también aquellos aspectos en los cuáles los especialistas son cercanos. Cabe destacar que el desarrollo de cada aspecto o categoría mencionada, no es una discusión acabada, sino que más bien, muestra los puntos relevantes y de discusión que los profesionales entrevistados señalaron como centrales, dichos aspectos se discutirán a futuro con el propio discurso de los jóvenes a los que se acceda como sujetos de la investigación.

Cabe destacar que para el análisis de la problemática será importante distinguir cuando se está hablando desde lo juvenil, los jóvenes, desde lo social o desde lo escolar.

\section{PRECISIONES CONCEPTUALES}

\section{Aproximaciones al concepto de exclusión social}

Cuando se plantea la pregunta por la exclusión social, en este caso los especialistas entrevistados, lo hacen acompañando el concepto de una segunda instancia interrogatoria ¿en qué sentido? ¿para quién? ¿según quién? ¿desde dónde?, la sola presencia de estas preguntas ya cuestiona y pone en tensión aquellas definiciones de carácter dicotómicas del concepto, es decir, «estar o no estar» dentro de algo, formar parte o no

Christian Berger, psicólogo, doctor (C) en psicología educacional, académico Universidad Alberto Hurtado.

María Teresa Rojas, profesora de historia y geografía, doctora en ciencias de la educación, académica Universidad Alberto Hurtado.

Luis Osandon, profesor de historia y geografía, doctor en ciencias de la educación, Director Departamento de Historia, Universidad Academia Humanismo Cristiano.

Raúl Zarzuri, sociólogo, magíster en antropología postítulo en investigación cuantitativa y cualitativa aplicada a la educación y las ciencias sociales, investigador CECS. 
o, tal vez, concepciones que sólo consideran como incluido o excluido, si se participa o no y en qué se participa.

En este sentido, lo primero que señalan, es que el concepto de exclusión social no puede ser visto sin su contrapunto, el de la inclusión social, de hecho, las políticas educativas más bien se refieren desde este concepto más que el de la exclusión. Lo anterior resulta relevante frente al intento de construir una definición, ya que al parecer no se está hablando de contrarios sino que de polos de un mismo continuo, dos énfasis de un mismo indicador o dos valores de una misma variable, es decir, se está hablando de grados de cercanía a uno de los dos polos. Nunca se está totalmente integrado a todo y nunca se está totalmente excluido de todo.

Un aspecto en el que convergen la mayoría de los discursos de los especialistas, es la necesidad de hacer la distinción entre una escuela que reproduce exclusión social, es decir, la escuela sería más bien un escenario social donde se manifiesta o se hace evidente la exclusión social, y otra donde la escuela en sí misma excluye. Esta última lógica sería más cercana a los planteamientos de Lumman respecto de los mecanismos de integración y los tipos de socialización dados en la escuela, donde la socialización se haría sobre un sujeto fragmentado, es decir, integración sistémica. Lo anterior, estaría indicando además la necesidad de precisar un contexto, un escenario y una temporalidad respecto del concepto en cuestión.

Hoy en día, en nuestro país, se declara la educación como central dentro de sus preocupaciones, en consecuencia, la entrada al sistema escolar como obligatoria, la cobertura escolar se encuentra en un $98 \%$ y la valoración social que tiene la educación dentro de la población es altísima (Dávila, entrevista personal, 2 de noviembre de 2007; Rojas, entrevista personal, 9 de noviembre de 2007). La Educación, es una tarea que no se cuestiona, ni desde el «deber de estudiar» ni desde el «querer estudiar», por lo tanto, cuando se analiza la exclusión social, debe ir más allá del fenómeno de ser partícipe o no de la experiencia escolar, si todos son incluidos, debe ser analizada desde otros ámbitos, desde otros parámetros. Al respecto, los especialistas coinciden en señalar que es la escuela, el espacio donde más incluidos se deberían sentir los jóvenes.

Otro aspecto importante señalado por los especialistas, es hacer la distinción entre lo netamente escolar y lo social. ${ }^{4}$ Una cosa es la ex-

4 No se están entendiendo como dos cosas distintas, sino que como ámbitos diferentes donde lo social en alguna medida incluye lo escolar. 
clusión social y otra, es la exclusión escolar, esta última da cuenta de aquellos estudiantes que son reiteradamente expulsados del sistema, aquellos que tras repetidas experiencias de fracaso escolar, sucumben en el sistema. Es en este sentido, que se podría pensar que las escuelas «malas» también son útiles (teniendo claridad que la calificación de «mala» resulta ambigua, ya que puede ser por rendimientos, por comportamiento, por perfil de alumnos $u$ otros factores además falta claridad) ¿por qué las escuelas tildadas de «malas» no desparecen? al parecer resulta necesario un espacio donde este tipo de estudiantes nómades puedan realizar su trayecto escolar (Rojas, entrevista personal, 9 de noviembre de 2007). Así mismo, «la escuela tiene una formas de estructurar el conocimiento y de transmitir el conocimiento que no tienen relación con las formas que actualmente los jóvenes están construyendo conocimiento» (Zarzuri, entrevista personal, 13 de noviembre de 2007; Osandon, entrevista personal, 15 de octubre de 2007). En este sentido, la escuela representaría un dispositivo de exclusión, en términos de no presentar una propuesta que les haga sentido a los jóvenes.

Lo anterior plantea la posibilidad de relacionar entonces la exclusión escolar a la deserción escolar, se podrían predecir perfiles de desertores, sin embargo, para este estudio, resulta interesante y necesario reflexionar sobre las consecuencias de la exclusión escolar más que en el perfil del desertor, las consecuencias del nomadismo escolar, consecuencias que se podrían ver transformadas en exclusión social, lo anterior dada la claridad que se tiene en Chile de lo que sucede si no eres parte del trayecto escolar obligatorio.

En este punto, cabe entonces hacer una precisión respecto de la investigación, esto es, dentro del fenómeno de la exclusión social, habría que ponderar a la escuela respecto de su «responsabilidad»o injerencia pero ¿cómo lograr ponderar? La respuesta a la pregunta, dentro de una investigación de carácter cualitativa, debería esperar el discurso del sujeto de estudio (joven escolar) en función de cuánto pondera a la escuela en esto, situándolo en la relación escuela-mundo social ¿debería la escuela hacer todo los esfuerzos para alcanzar la inclusión social de todos los jóvenes? ¿en qué sentido? ¿en qué consistirían estos esfuerzos? Finalmente, entonces, cómo se podría entender la relación entre exclusión social y exclusión escolar.

Es sobre lo planteado anteriormente, donde se debe hacer otra distinción. Hablar de exclusión social en el contexto escolar o educativo, implica distinguirlo también de conceptos como el de la discriminación. La experiencia indica, que la discriminación en la escuela se 
relaciona con recalcar negativamente la diferencia de algunos estudiantes: por ejemplo, por ser malo para matemáticas, por tener un rendimiento más bajo, por tener sobrepeso, por ser más solitario, entre muchos otros ejemplos. La discriminación de todas maneras marca un acto en desmedro de alguien, sin embargo, no necesariamente implica exclusión social. Puedo ser discriminado en la escuela por «flaite»o por «nerd» situaciones intraescuela, lo que no significa que no entre a la universidad a estudiar, que implica ir más allá de ella. Como lo plantean Antivilo, Cavieres y Valdivieso (2006) «la exclusión social es una forma de discriminación, pero, trasciende el acto discriminatorio ya que sus implicancias afectan al propio ordenamiento social» Entonces, cuando se habla de exclusión social, va más allá de lo que se podría entender como discriminación, es un fenómeno donde la escuela se encuentra involucrada por ser parte del sistema social más amplio, aquí entonces la pregunta ¿cuándo una escuela posibilita o potencia la exclusión social? o ¿cuándo una escuela posibilita la integración social? y si el sujeto se considera discriminado al interior del establecimiento en el que participa ¿qué es lo que hace la escuela para lograr que ese sujeto reafirme que no es la escuela un lugar que le represente utilidad o significado para su proyecto de vida?

En definitiva la exclusión social, debe ser entendida en grados, niveles y relacionada a un contexto particular, así lo plantean Antivilo, Cavieres y Valdivieso (2006) cuando señalan que la exclusión social se puede entender como un fenómeno relacional, subjetivo, contextual y temporal, cuyo análisis o evaluación se precisa desde las diferentes áreas que conforman lo social. Lo anterior, si se piensa en la escuela o el ámbito educativo de la sociedad, debe ponderarse en función de un sistema más amplio.

\section{2. ¿De qué se compone la exclusión social?}

Al respecto, los especialistas entrevistados señalan que el joven de enseñanza media, tiene una alta valoración respecto de lo que significa estar en el liceo. Lo anterior, dado que representa uno de los espacios fundamentales de inclusión social para ellos(as), independiente de las condiciones de calidad de la enseñanza, éste es un espacio donde se encuentran con pares, con referentes y cumplen con la trayectoria obligatoria, una trayectoria que estructura gran parte de nuestras vidas, la trayectoria escolar. Es sabido que cerca del $98 \%$ de los jóvenes en edad escolar asisten a la escuela, es decir, el problema de la cobertura 
escolar, al ser superado, implica que la exclusión social, ya no se trataría de «estar dentro o fuera» del sistema escolar, tendría entonces otros matices de mayor complejidad, como los que plantea Dávila (entrevista personal, 2 de noviembre de 2007) que están en relación a los capitales heredados, acumulados y escolares con los que cargan los jóvenes actuales y cuánto estos pesan en sus proyectos futuros, en el diseño de sus trayectorias después del egreso del Liceo, esto es, cuánto significan un límite mínimo que deben superar, un límite del que no pueden escapar o un horizonte de oportunidades y metas.

A lo anterior, Dávila, Ghiardo y Medrano (2005) agregan que ésta generación:

Es una generación que está contenta con la vida que lleva y manifiesta un tremendo optimismo en su futuro y en su vida futura, que adopta un sentimiento de pragmatismo, ven de manera práctica y vivencial si sus expectativas optimistas tienen un correlato con sus experiencias de vida. El problema es que sospechan del desajuste entre esas altas aspiraciones y expectativas, las que ven difícil de concretar, reconocen que tienen pocas posibilidades para cumplirlas, por los desiguales accesos a las oportunidades sociales, de acuerdo a los orígenes sociales familiares, los bajos capitales heredados y la escasa movilidad social que estaría ofreciendo la sociedad chilena, resultando complejo el compensar o revertir las desigualdades de origen (p. 279).

Este desajuste de expectativas y oportunidades antes mencionado, da cuenta que si la institución escolar representara un dispositivo de exclusión social, este se manifestaría posterior al egreso del Liceo, es decir, representaría más bien un «pre-antecedente», antecedente a otro proceso relacionado al mundo productivo. En este sentido, Ghiardo y Dávila (2007) señalan:

Las distintas dimensiones de la integración social de los jóvenes han estado en el centro de las preocupaciones sobre lo juvenil. Las dimensiones básicas para medir esta integración funcional han sido la educación, la salud y la integración al mundo del trabajo. La importancia de esta última dimensión es incuestionable, sobre todo por el peso que tiene en la configuración ideal y práctica de los proyectos de vida de los jóvenes. No hay que olvidar que el tránsito hacia la vida productiva es uno de los elementos decisivos en la construcción de la juventud como categoría social (p. 27). 
Al respecto Redondo (2000) caracteriza la situación actual como un escenario complejo donde se interceptan 3 ámbitos que dan cuenta de capitales diferentes dentro de lo social: el escenario tecno-económico, que se refiere principalmente al capital financiero, su estructura y forma de funcionamiento; escenario socio-politico, que se refiere a los espacios de participación pública y política, a los grupos de poder $y$ de opinión; por último el escenario cultural-ideológico, que se refiere a la plataforma valórica donde se mueven y se motivan los individuos para su accionar. Dicha caracterización, lo que señala es que la relación entre la escuela, la educación y los proyectos de vida de los jóvenes, tiene una complejidad que sobrepasa lo propio de la escuela, sus muros, toca directa o indirectamente la oferta y el sentido de la educación para los jóvenes, específicamente «de la educación que reciben, refiriéndose al tipo de educación que reciben», en este sentido, el fenómeno de la exclusión social relacionada a la escuela, tiene como plataforma esta complejidad.

Lo anterior, orienta a pensar entonces que cuando se habla de exclusión social desde los escenarios educativos, estos inevitablemente están relacionados con las trayectorias de vida de los jóvenes y sus proyectos de vida y cómo interviene la experiencia escolar en esto, esto implica asociarlo al mundo productivo, al mundo del trabajo, al mundo de la participación y en consecuencia al valórico.

Tal vez, podría resultar interesante agregarle un indicador de carácter subjetivo que hablara de la satisfacción del trayecto y proyecto de vida, le daría un matiz a la exclusión social, o más bien genera la pregunta ¿desde quién se debe mirar el fenómeno de la exclusión? desde quien dice que se siente excluido o se percibe a sí mismo como excluido o incluido socialmente, o desde ciertas categorías preconcebidas que dada su existencia, presencia y grado de presencialidad sería o no una persona excluida o incluida socialmente. Pensando en la diversidad cultural, en la globalización, la diversidad valórica, la declinación de las instituciones y sus clásicos discursos éticos y morales totalizadores, ya no resulta tan claro en qué sentido se incluye a un sujeto dentro de la sociedad; esto resulta tener una diversidad mucho más amplia, por lo tanto también se tensiona el aprendizaje social dentro de la escuela, se tensiona en algún sentido su finalidad. Sobre estos argumentos, es que la evaluación de la inclusión o la exclusión, debe tomar en cuenta el carácter subjetivo de quien se declara incluido o excluido, es decir, de su experiencia. 
En este sentido, lo que plantean Antivilo, Cavieres y Valdivieso (2006) agrega respecto de este desfase anteriormente mencionado, entre aspiraciones, expectativas de éxito y percepción de oportunidades reales de alcanzarlo, produciría una suerte de «tensiones existenciales y le proporciona la dimensión subjetiva al concepto de exclusión» de alguna manera es el indicador y el componente de este concepto que aporta a la respuesta de la pregunta ¿desde quién? ¿para quién? A modo de ejemplo, tal vez es el mismo joven quien no quiere participar, sintiendo que el ser excluido de ciertos espacios le acomoda y lo identifica, por otro lado, como lo desarrolla Dávila (entrevista personal, 2 de noviembre de 2007) esta dimensión subjetiva de la exclusión social se podría ver reflejada ante el hecho de que muchas veces es el propio joven quien se autolimita, esto es, a propósito de sus marcas de origen como lo llama el autor, el joven no se sitúa en un escenario que le resulta adverso, reafirmando un sentido incluso identitario a partir de la diferencia. Es decir, los parámetros subjetivos se asocian a la construcción de identidad y a los referentes identificatorios de los jóvenes.

Por su parte, en la búsqueda de precisar el concepto, agrega Rojas (entrevista personal, 9 de noviembre de 2007) que la pobreza ya no sería un indicador de exclusión social, sino que más bien, haciendo la distinción entre exclusión social y exclusión escolar, el «excluido» del sistema escolar, es aquel que no responde a los parámetros de comportamiento y de rendimiento establecidos por la institución, los que no necesariamente se asocian a la pobreza., se manifestaría en una suerte de «síndrome de nomadismo escolar», donde el posible desertor del sistema escolar, vagabundea entre muchos establecimientos e incluso de diferentes comunas, para finalmente ser un sujeto poco deseado en la escuela, un sujeto del que ya no se quiere hacer cargo, en definitiva, con igual nivel de pobreza hay quienes se excluyen y quienes se quedan.

En este sentido, los especialistas coinciden en decir que las escuelas son más bien homogéneas en su interior, que inevitablemente, el sistema escolar está segmentado, está ordenado según estrato socioeconómico, lo que hace que entre escuelas la cosa sea diferente; más que tener diversidad en su interior hay diversidad entre las escuelas, en palabras de Baeza (entrevista personal, 10 de octubre de 2007),

Las escuelas más que tener diversidad en su interior, hay mucha diversidad entre las escuelas, no es esa escuela republicana francesa o la escuela republicana chilena de décadas atrás donde en el mismo lugar 
existía un conjunto importante de jóvenes donde estaba desde el hijo del médico del pueblo, hasta el hijo de la empleada del pueblo, eso ya no es así; ahora hay escuelas diferentes para ambos tipos de «hijos de»; eso no significa que al interior de esta escuela fragmentada - que tiene cierta homogeneidad en su interior también - no haya heterogeneidad, es decir, son conglomerados de heterogéneos entre sí, pero tampoco homogéneos en su interior; hay más heterogeneidad entre las escuelas municipales con respecto a las particulares pagadas o subvencionadas, y al interior de ese segmento un poco de homogeneidad.

La construcción de sentido de los jóvenes va variando de un joven a otro, perteneciendo casi todos a la misma generación, al mismo establecimiento, al mismo curso. De aquí entonces, ante la igualdad de condiciones dentro de un establecimiento, cabe preguntarse ¿quiénes tienen experiencia de exclusión social?

Por otro lado, Osandon (entrevista personal, 15 de octubre de 2007) plantea indicadores de exclusión, o más bien factores causantes que tendrían como consecuencia la exclusión social de alguna forma, esto teniendo en consideración que hay que diferenciar aquello que es exclusivamente exclusión escolar de aquello que es social. Dichos factores estarían en el orden curricular y pedagógico de la escuela. Frente a lo anterior, el especialista plantea que al no representar la escuela hoy en día, lo que representaba hace varias décadas atrás, una escuela que representaba movilidad social, la pregunta no está en cuantos recursos recibe la escuela, sino de cómo esta escuela se entiende a sí misma en contexto de exclusión social. La educación intentó dar un cambio integrando la clave de la diversificación, es decir, las escuelas deberían saber que hacer en el contexto que les toca desempeñarse, en este sentido, la educación ha ido transitando progresivamente a una clave de homogenización curricular, es decir, todos los espacios de apertura curricular se han ido cerrando y reestandarizando vía indicadores de rendimiento en general y de desempeño del profesor.

Así los acentos de la escuela no están puestos en la diversidad, en la cultura juvenil por ejemplo, sino que más bien en el cómo se rinde en el SIMCE. Estos últimos, parámetros de rendimiento, se han ido transformando en criterios de calidad, lo que implica que las estrategias y los esfuerzos de la escuela hacia la atención a la diversidad e inclusión sean menos relevantes, o sea bajan en prioridad. De esta forma, resulta entonces coherente pensar que la escuela hace lo que está a su alcance, sólo lo que puede hacer en cuanto a los jóvenes y 
sus espacios de expresión y desarrollo, lo que tal vez no es necesariamente lo que para los jóvenes apoyaría procesos de inclusión.

Transformar este aspecto en indicadores, tendría tal vez que relacionarse cuando los jóvenes hablan de los sentidos de la escuela, del sentido de lo que aprenden en su interior, cómo construyen sentido e identidad en el espacio escolar y cuánto la escuela aporta en esto y en cómo lo hace.

En definitiva, los indicadores de exclusión social, aunque parezca obvio, dependerían de lo que se entendería por exclusión social, del lugar desde donde se lo quiera mirar; ya que hasta ahora, lo que se podría ir asumiendo como supuesto de investigación, es que la exclusión social, se relaciona con muchos ámbitos, tiene niveles, tiempo y contextos. En rigor, se podría decir que la exclusión social no existe ya que siempre se está en alguna parte, siempre se pertenece a alguna categoría social, a un espacio social a una temporalidad, sin embargo, no siempre corresponden con la ideología imperante, organizadora o estructurante de la época.

\section{TEMAS DE REFLEXIÓN}

En este punto, se dará cuenta de aquellos ámbitos temáticos y categorías que se rescatan de los discursos de los especialistas. Son puntos de tensión que pretenden ser comparados con los discursos de los jóvenes en la investigación en la que decantará esta primera parte.

\section{1. ¿Cuál es la promesa de la escuela?}

La pregunta apunta a cuestionar cuáles son las ofertas que hace la escuela, cuál es la articulación de esta trayectoria escolar y la que se debe construir después de ésta el joven ¿qué se lleva de la experiencia escolar, qué interpretación hace? Como ya se ha planteado anteriormente en este trabajo, la escuela ya no representa un camino para la movilidad social. En este sentido, el joven encuentra con facilidad otros espacios que le son más útiles al momento de hablar de posibilidad de acumular capital cultural y capital de consumo. Si bien se valora la escuela, se sabe lo importante que es pasar por esta instancia, el problema es que no quieren «esa escuela»; así estructurada como está, no es lo que aspiran como educación. Al respecto Dávila, Ghiardo y Medrano (2005) señalan que, 
La escuela no logra aún reconocer el universo aspiracional que plantean sus alumnos, sus altas expectativas y metas educacionales; reconociendo sus pocas posibilidades. La escuela se ha centrado en el terreno de las posibilidades de sus alumnos. La administración de las aspiraciones se ha orientado y fundado con base en un diagnóstico e imagen errada sobre su alumnado, no en el plano de ampliación de aspiraciones, sino que más bien en su acortamiento, bajo el apelativo de las - pocas- posibilidades reales de concreción» (pp. 281-282).

Por su parte, Berger (entrevista personal, 9 de octubre de 2007) señala lo anterior, apelando a una suerte de «desesperanza del sistema escolar», cuestionando la oferta de la escuela, ésta demanda el cumplimiento de un perfil de estudiante, de un perfil de ciudadano y la pregunta que le sigue a esto es el ¿para qué? La escuela, incluye a los jóvenes, pero en términos nominales, produciendo finalmente los llamados caminos paralelos, las socializaciones paralelas o contra la escuela, dado que simbólica y culturalmente los deja fuera, los excluye.

Se sabe bien lo que pasa si no se estudia, pero no se sabe que pasa después de la escuela. Hay un problema de articulación entre expectativas y aspiraciones de los jóvenes y el sentido de la escuela (Dávila, entrevista personal, 2 de noviembre de 2007; Zarzuri, entrevista personal, 13 de noviembre de 2007; Osandon, entrevista personal, 15 de octubre de 2007; Rojas, entrevista personal, 9 de noviembre de 2007) Los jóvenes al no sentirse integrados, buscan otras integraciones, integraciones de carácter más pequeñas, pero en espacios o ámbitos donde sí se les da respuesta y se les presenta utilidad para moverse en esta sociedad.

Los jóvenes de hoy son diferentes, e incluso entre los jóvenes la diversificación es masiva. Ellos se construyen en un escenario social, cultural, económico diferente, lo que implica que la escuela debe romper con la pretensión de continuidad de lo que se venía ofreciendo, lo que se vive hoy en día, en palabras de Zarzuri y Ganter (2005) es justamente una «ruptura generacional» y es sobre este escenario que la escuela debe pensarse a sí misma en un escenario diferente, su promesa debe ser reformulada.

\section{Sentido de la escuela}

La escuela, ya no representa el sentido moderno, que prometía y generaba movilidad social. La escuela debe ponerse a sí misma en un nue- 
vo escenario social y dentro de éste, en escenarios de exclusión social. Para el joven entonces cabe la pregunta ¿cómo me construyo como sujeto al interior de la escuela? ¿cómo construyen el sin sentido dentro de la escuela? Al respecto Osandon (entrevista personal, 15 de octubre de 2007) señala que cuando se piensa en aprendizajes significativos, que podría implicar para los jóvenes sentimiento de inclusión; el especialista señala que si bien es un concepto fuertemente instalado, lo objetiviza a su vez como deslavado de sentido. Esta no pasaría por una reflexión de carácter cognitiva, es fundamentalmente sociocultural, antropológica, económica, es decir, se relaciona con el «otro» con la «alteridad» con el sujeto que el profesor tiene en frente, un sujeto con biografía, historia, familia, pares, estética. Este sujeto entra con todo esto a la sala de clases, en palabras de Dávila, entra con capitales, que no necesariamente son considerados dentro de la sala de clases, dentro del proceso de enseñanza aprendizaje.

El profesor, debería tener noción de esto, debería tener contemplado ese otro, un sujeto que ingresa con biografía, con contexto, con familia, con pares, ingresa con todo eso a la sala de clases. Sin embargo, lo anterior hay que tomarlo en un contexto de 30 ó 40 alumnos, que son todos otros, y estos otros en este contexto es aproblemante, ya que las expectativas de ingreso a la escuela, no tienen que ver con que es lo que voy a entender y que es lo que me va servir para la vida probablemente en una primera instancia. Aquí está la tarea del profesor, acompañar el proceso de construcción de sujetos al interior de la escuela. Esto es, enseñar contenidos es una parte de la tarea, también es importante entender que hay otro que espera ciertas cosas, que tiene expectativas, en definitiva, entender que son jóvenes que funcionan con un sí mismo, con un entorno y que desde esos pisos, emerge lo que a él le puede interesar de la historia, de la matemática, del lenguaje. Claramente, lo anterior requiere mucho trabajo de preparación de la acción concertada en la escuela. En este sentido, una educación inclusiva significa acoger al otro en sus diferencias, pero es una diferencia que también tiene que ser negociada con la compulsión de la enseñanza escolar obligatoria (Osandon, entrevista personal, 15 de octubre de 2007).

Así mismo, Zarzuri (entrevista personal, 13 de noviembre de 2007) agrega «lo que le ofrece la escuela a los jóvenes en la jornada escolar completa no es precisamente lo que ellos demandan, sino que más bien hacen aquello para lo que se sienten habilitados, lo que pueden hacer, nada más que eso», "la escuela hace una oferta que no interpela a los jóvenes» (Zarzuri, entrevista personal, 13 de noviembre 
de 2007; Berger, entrevista personal, 9 de octubre de 2007; Osandon, entrevista personal, 15 de octubre de 2007), en este plano y considerando la alta confiabilidad y valoración que tiene la educación al interior de la población chilena, surge entonces la tensión respecto de que «no es que a los jóvenes no les interese la escuela, no les interesa esa escuela» no les interesa la escuela como está estructurada hoy, el funcionamiento, los productos esperados de la escuela, las metas. De alguna manera es lo que aleja a los jóvenes de la escuela y en general de la institucionalidad, el joven se rebela contra ella y crea socializaciones paralelas.

Bien lo señala o ejemplifica Salazar (2006) a propósito de la exclusión social y la pobreza:

A diferencia del siglo XX, en que se comenzaron a desarrollar movimientos sociales abiertamente antioligárquicos, que pasaban inclusive por desconocer de cierto modo la legalidad pública y proponer otra legalidad y otra constitución política, hoy en día no se es plenamente consciente de la desigualdad. El problema se vivencia fundamentalmente como un fracaso personal frente al mercado de trabajo o frente a tu familia. Se subjetiva el conflicto, y se traduce en agresión en el entorno, hacia los hijos, hacia el cónyuge. Por último es una violencia que va de lo doméstico a lo social. Y todo eso se traduce en definitiva en distintas formas de delito. En consecuencia, no tiene una configuración en términos políticos. Sin embargo, la juventud popular que padece la pobreza está haciendo una traducción política indirecta. La reacción popular juvenil culturiza esta situación de pobreza, y la transforma en lo que se llama la estética del descontento, o la cultura de la rabia. Eso es una primera fase de la transformación de la frustración en una expresión política, pero no quiere decir que estén ya politizados.

Además, relacionando lo anterior al movimiento secundario, el mismo autor responde:

Esta reacción juvenil se ha dado, curiosamente, más en el sistema formal de enseñanza secundaria que en la universidad [risas]. Sobre esto, tengo una hipótesis. Toda la crisis del sistema, todo el impacto real de esta desigualdad en la distribución del ingreso lo reciben los niños. Los niños tienden a reaccionar frente a toda esta crisis desarrollando dos tipos de conductas: uno, es una conducta solidaria, en que los niños atraen una solidaridad de los jóvenes adolescentes organizados. Y luego, atraen a los viejos de la población, dirigentes de clubes deportivos, centros de madres, generando en torno suyo un ambiente y una cultura 
local de solidaridad. Por otra parte, la frustración que les llega por el lado de los papás que no tienen pega, que son borrachos... les hace desarrollar actitudes violentas. Si juntas las dos patas, solidaridad y rebelión violenta, eso configura inmediatamente el sujeto revolucionario. Eso es lo que ocurrió con el movimiento secundario. La solidaridad se expresó en la anchura del movimiento, en la idea de la asamblea, en la soberanía que ésta ejerce, ligado al mandato que le dan a los voceros. Pero la rabia también se expresa en la forma en que se manifestaron en las calles.

En Chile, la $4^{\text {a }}$ Encuesta Nacional de la Juventud del Instituto Nacional de la Juventud (INJUV), que se aplicó a una muestra de más de siete mil jóvenes, entrega algunas pistas en ese sentido: entre los $15 \mathrm{y}$ 29 años son críticos respecto a la democracia, pero no creen que haya otro sistema mejor; exigen más igualdad de oportunidades, pero no eligen autoridades que los representen; son individualistas, pero participan en organizaciones solidarias (voluntariados) y no se identifican con ningún partido.

Hay un distanciamiento creciente de los jóvenes con los procedimientos de la política formal. El 13,2\% confía en los parlamentarios y sólo un $7,3 \%$ en los políticos, pero esto no ha sido un obstáculo para que un número cada vez mayor valore la democracia. Más del 60\% está de acuerdo con este sistema, pero creen que hay que perfeccionarlo. La falta de oportunidades y la desigualdad social son los grandes problemas que ellos detectan (INJUV, 2000).

Su herramienta de combate no son las instituciones tradicionales como la iglesia, la familia, la política, que son justamente las que pueden generar políticas para dar respuestas más efectivas. Sus esfuerzos están puestos en las organizaciones sociales o de voluntariado de elección propia, o incluso en la acción callejera violenta a través de grupos de autoidentificación anarquista.

En este sentido, no se refiere a que los jóvenes no quieran participar, sino que más bien se rebelan ante las formas de participación que se les propone.

La política, por ejemplo es una cosa de no conexión con el tema juvenil... A ese nivel los chiquillos dicen no, a nosotros no nos interesa esa política, a nosotros no nos interesa esa escuela - no les interesa esa escuela-; seguimos siendo creyentes, sí po', seguimos creyendo en la política; sí, también, seguimos creyendo que hay que educarnos, tam- 
bién y de hecho es esa la apuesta (Zarzuri, entrevista personal, 13 de noviembre de 2007).

Por último, cabe destacar que la trayectoria de los jóvenes ya no es lineal, por lo tanto la oferta de la escuela debería ser igualmente diversa, contemplar la diversidad, la heterogeneidad dentro de la igualdad de condiciones. Al respecto fueron varios los especialistas entrevistados que pusieron hincapié, como Berger (entrevista personal, 9 de octubre de 2007) en que la escuela funcionaba, priorizaba y proponía a sus estudiantes desde una lógica adultocéntrica, no desde una lógica de las juventudes, ya que no es una juventud sino que varias juventudes, por lo tanto, ellos comienzan a desarrollarse en espacios paralelos, teniendo socializaciones paralelas o contra la escuela. Los jóvenes tienen mayores posibilidades sobre la cual construirse, no solamente la escuela o el tipo de escuela chilena.

\section{La exclusión social y su relación con la pobreza}

Hasta este punto, se han realizado precisiones conceptuales, las que podrían implicar consideraciones metodológicas para una investigación en este ámbito. Uno de los aspectos que se imprime en este trabajo con claridad es la necesidad de reformular el concepto de joven, joven escolar, y exclusión social, y que pensar el estudio desde «solamente» la pobreza sería entonces problematizar poco lo que se quiere estudiar.

Son múltiples las exclusiones o no inclusiones asociadas a la escuela, pero no necesariamente es porque la escuela las genere, sino que más bien es un fenómeno cultural que está asociado al mundo adulto, al mundo considerado productivo, aquel que le es útil a la sociedad. A los jóvenes no se les dan esos espacios de poder, desde donde se puedan interesar por la educación, la política, la religión, su participación se relaciona con otros espacios. Como se dijo anteriormente, al joven no es que no le interese la escuela, no está interesado en «esta escuela», la actual; aquí es donde se hace más claro el hecho de que estamos hablando de un concepto de exclusión social de carácter relacional, es decir, tiene que ver con otros grupos, está en relación a otros. Siendo así, los sujetos se encuentran más cercanos a ciertos grupos y más lejanos a otros grupos; entonces el concepto se refiere a un continuo que va desde la completa inclusión a la completa exclusión y se evalúa en relación a otros; esto es, no necesariamente por la presencia o ausencia de unos factores predeterminados, como el de 
más o menos educación familiar, si bien es un referente, éste resulta complementario.

Lo que aportaría poder mirar la exclusión como un fenómeno más global, cultural y no sólo desde la pobreza, sería tener la posibilidad de dar cuenta y distinguir los estigmas que se le adjudican a la pobreza, a la juventud y a la asociación de ambos; daría la posibilidad de ampliar el espectro y realizar una investigación que recupere conceptos, como el de diversidad e inclusión, pero no pensando solamente en políticas compensatorias o focalizando los esfuerzos en las escuelas de funcionamiento y rendimiento más precarios.

Si bien en cierto que todos podemos ser y somos, en algún sentido 'extraños', más aún en nuestras grandes ciudades, es preciso que las ciencias sociales se cuiden de agregar al estigma 'extraño', adjetivos tales como 'peligroso', que implican y conducen a muchos jóvenes a una suerte de 'exilio cultural', al cual se suman los 'exilios' económicos, sociales y políticos (Zarzuri y Ganter, 2005).

\section{Homogeneidad interna $v / s$ heterogeneidad externa en las escuelas chilenas}

Respecto al anterior punto desarrollado - y como ya se ha hecho mención- un punto central es que las escuelas en su interior son más homogéneas que heterogéneas, más bien entre las escuelas hay una suerte de segmentación por estrato social y, «lamentablemente» en Chile, esta estratificación se ha transformado en una especie de indicador de calidad: mientras más pagas por la educación más calidad se tiene asegurada. Si bien éste fue un tema planteado, sin desconocer la diversidad dentro de los jóvenes, ¿cuáles serían los criterios que el joven consideraría que la escuela utiliza para clasificarlos, considerarlos, incluirlos o discriminarlos? ¿cuál es el joven que debería ser sujeto de estudio de la investigación?, al parecer, no necesariamente pobre.

Si bien resulta interesante la diferencia por estrato socioeconómico, el concepto de exclusión social que finalmente adoptará la futura investigación, más bien se refiere a un fenómeno relacional y subjetivo en que los escenarios de exclusión podrían ser variados; dentro de los cuales podría estar la pobreza, pero no necesariamente como único parámetro.

En este sentido, las escuelas sufren una suerte de segmentación social. Existe una distribución innegable en la que las escuelas públi- 
cas catalogadas de menor calidad académica, atienden a estudiantes de niveles socioeconómicos bajos. Esto es, la distribución de las escuelas en función de su calidad académica es directamente proporcional al nivel socioeconómico de las familias y del sector, la segmentación social coincide con la segmentación de las escuelas. Siendo así, las posibilidades de instalar procesos de socialización orientados a la integración social, quedan cerrados en la misma escuela, cada escuela lo resuelve según sus posibilidades, sus recursos, su entorno y se diluye en relación a las otras escuelas, no hay una intervención u orientación al cambio de carácter más político, social. Aquello implica que cada establecimiento perpetúa su situación.

La escuela en sí misma, en su interior, tal vez no reproduce una estructura social, sino que más bien lo haría en función de que su finalidad y la trayectoria al interior de ésta, se encuentra en relación a algo, no es por sí misma, sino que está en relación a otros establecimientos con otras características sociales, demográficas, culturales, entre otras. A su vez que se encuentra en relación a un sistema más amplio, es parte e él.

\section{Fragmentación, segmentación y estigmatización}

Pensando en las políticas que buscan generar mayor equidad e inclusión a través de la educación; aparece una fuerte crítica a éstas, por el hecho de ser políticas que muchas veces lo que generan es mayor fragmentación o una suerte de mayor estigmatización de aquellos establecimientos con mayor necesidad de focalización de recursos, ya sea económicos, tecnológicos u otros (Baeza, entrevista personal, 10 de octubre 2007). En este sentido, se podría decir que aumentan la segmentación, lo que va en desmedro de la posibilidad de inclusión social en el contexto escolar. Estas políticas, resultan ser, en palabras de Osandon (entrevista personal, 15 de octubre de 2007) políticas compensatorias, focalizando mayores apoyos desde el punto de vista de los recursos para la escuela. Sin embargo, si se requiere pensar en que la inclusión social, pasa por un ámbito simbólico, cultural, no sería la focalización de recursos económicos lo necesario para alcanzar mayor inclusión social de los estudiantes desde las escuelas. Por ejemplo, aquellos establecimientos que son rotulados desde el ministerio como establecimientos focalizados, están indicando que son instituciones con necesidades y con resultados académicos que están muy por debajo de la media, estas son políticas de discriminación positiva, 
que de todas maneras implica una estigmatización negativa detrás, hay una imagen negativa que se acompaña con la política.

\section{Jóvenes e institucionalización}

En general, investigaciones a nivel mundial — como la de Imaz (2002) en España-, las que se acercan a caracterizar y conocer el mundo juvenil desde el plano de sus valorizaciones u orientaciones de acción, hablan de ellos(as) como un grupo donde prima un relativismo moral, es decir, donde las reglas, las normas y las valorizaciones no tienen un carácter definitivo, estable o absoluto. Existe un talante o actitud frente a ciertos temas valóricos que van desde la pasividad, lo acomodaticio, la indiferencia a la actividad y al compromiso; sin embargo, las adhesiones se acompañan de emociones de apego y desapego, por ejemplo «está de moda defender los derechos humanos» ¿es un principio de vida adoptado, o es un principio circunstancial que permite la pertenencia a un grupo? En este mismo sentido, otros estudios, concluyen que los jóvenes le dan cada vez menos importancia a todo. La tendencia observada es el abandono de todo ámbito de trascendencia, sobre todo el político y el religioso; la gran importancia a la amistad, menos asunción de riesgos y menor contacto con el exterior. En su vida (fragmentada y deconstruida) priman la simultaneidad y la superficialidad por un lado, la atomización y la economía de la acción por otro.

Lo anterior implica que - respecto de los agentes tradicionales de socialización - los jóvenes actuales adoptan un actitud de recepción distante; los jóvenes deconstruyen y reconstruyen, desde sus experiencias lo que los agentes tradicionales de socialización les transmiten. La ausencia de modelos exteriores claros, el surgimiento de una diversidad cada vez mayor de éstos y la constante experimentación hace que, a la postre, el último referente en la construcción del puzzle es el propio joven, según las cosas le vayan bien o no al propio joven (Imaz, 2002).

Al respecto Zarzuri (entrevista personal, 13 de noviembre de 2007) señala que entre los jóvenes hay una distancia que cada vez crece más respecto de la institucionalidad pública y privada. No es de extrañar lo dicho anteriormente, síntoma de aquellos, es el hecho que si bien la generación actual es la que tiene más alta escolaridad en la historia de Chile, sin embargo no ha podido construir espacio, disputan el poder al interior de los espacios sociales donde se mueven, como por ejemplo la escuela, de aquí la clásica tensión entre cultura 
juvenil y cultura escolar. Esto es a lo que el sociólogo francés, Francois Dubet, se refería con la declinación de la institución.

El autor plantea que las instituciones sociales ya no representan los valores centrales, orientadores de acción, es un tema que se traslada cada vez a un espacio más pequeño el cara a cara.

Durante los últimos 30 años, la imagen de las instituciones ha estado profundamente desestabilizada (Dubet, 2002).

Desde hace medio siglo, las instituciones han estado sometidas a dos críticas: la primera, reposa a la vez sobre una reificación del modelo institucional, reducido a una voluntad de poder y de normalización. El programa institucional ha salido desencantado, sus ideas de educar han estado reducidas a la dominación y al poder. La imagen del hospicio y de la prisión se ha impuesto como aquellas de la forma pura y de la naturaleza profunda de las instituciones de socialización. Más liberal y más política, la segunda crítica está puesta sobre otro registro. En una sociedad en constante cambio y en constantes marchas de nuevas demandas sociales, las instituciones aparecen como entes rígidos, de mundos cerrados, de una centralización del Estado (Dubet, 2002).

\section{ELEMENTOS PARA LA INVESTIGACIÓN}

En este apartado, se hará mención de ciertos elementos que precisan de alguna manera la problemática, los objetivos, el objeto de estudio y los casos del mismo. Sin embargo, cabe destacar que se presentan a modo de reflexión, esto no implica continuar precisando y ajustando, sobre todo, la metodología del estudio y el uso de estudios complementarios ya realizados.

El fenómeno de la exclusión social, en este caso específico, será enfocado como un fenómeno relacional y subjetivo, contextual y temporal que se podría entender desde diversos ámbitos que conforman lo social. Esto significa, que el análisis del discurso que se realizaría necesariamente siempre tendrá que considerar respecto de qué y de quién aparece el sentimiento de exclusión social, es aquí donde hace evidente la consideración de la exclusión social como un fenómeno relacional, esto es, alguien o algo excluye a «otro», alguien que no se ajusta al perfil o a las expectativas necesarias para ser incluido. Así mismo, considerar como construyen este sentimiento apuntando al proceso, cuáles serían esos dispositivos y si consideran que éstos están instalados en la escuela. 
Por un lado, la escuela como espacio socializador legitimado por la sociedad, no es ajena al escenario social más amplio, que actualmente, se caracteriza por la incertidumbre, la globalización, la inmediatez, entre otras cosas. Dicha situación, a la vez, ha traído consigo nuevas formas de exclusión social, ${ }^{5}$ espacios donde los jóvenes escolares se desenvuelven en busca de oportunidades y en busca de construcción de identidad y de sujeto en definitiva. Por otro lado, los jóvenes por el hecho de vivir en esta etapa de desarrollo (adolescente), cruzan por cambios importantes, cambios que traen crisis, y principalmente, resistencia a lo que el mundo adulto les propone incorporarse. Dicha situación, para la escuela y para las políticas educativas, ha significado tener que ir reflexionando y replanteando sus objetivos, sus metas para que tengan sentido frente a los grandes cambios que va sufriendo la sociedad en general. Entender la exclusión social desde el discurso de los jóvenes, tal vez aportaría a entender o entregar luces a las políticas educativas de inclusión social. En especial, pues el aporte estaría en tomar en cuenta que el fenómeno en cuestión se produce no sólo por condiciones objetivas a las que generalmente apuntan las soluciones en política pública, sino que también apuntaría a una construcción subjetiva del beneficiario directo de los establecimientos educacionales, terreno que tal vez no ha sido considerado en dichas planificaciones.

Tomando en cuenta lo anterior, los jóvenes, han hecho fuertes críticas a la educación chilena; de hecho, una de las manifestaciones que quedarán guardadas en la historia de la educación en este país, ha sido el movimiento estudiantil secundario del 2006; en el que parte de la fundamentación de su lucha, tiene relación con la fuerte crítica que le hacen a la educación respecto de la calidad, la pertinencia y la equidad que ésta ofrece (CESC, 2006).

Por su parte, el Ministerio de Educación chileno ha puesto en marcha una serie de programas y proyectos focalizados en aquellos establecimientos de mayor vulnerabilidad y de peores resultados académicos. Así mismo, ha puesto en marcha iniciativas como la incorporación de las cuotas obligatorias de alumnos(as) vulnerables en la educación subvencionada, políticas que han funcionado como políticas compensatorias, las que muchas veces han generado mayor distancia entre escuelas que mayor integración social. Sin embargo, el sujeto

5 La urbanización, el poder de la comunicación cibernética, el Transantiago han traído nuevas formas de marginación de la población. 
de este estudio, debe ir más allá de una mirada sólo desde la pobreza, pensando en que la pobreza por excelencia ha sido uno de los escenarios más asociados a la baja calidad escolar. Resulta importante, no sólo considerar variables objetivables, discretas u otras preestablecidas; sino que también resulta importante y complementario, considerar el ámbito subjetivo del fenómeno de la exclusión social

Tomando en cuenta estos antecedentes, con esta investigación se espera comprender desde el discurso de los jóvenes que cursan enseñanza media, en el trayecto escolar donde se van construyendo como sujetos. Lo anterior se articula en preguntas tales como: ¿aparece el sentimiento de exclusión social? ¿en qué sentido? ¿sobre qué escenarios? ¿en qué medida la escuela aporta a que se construya dicho sentimiento? Por otro lado ¿sería el mismo sentimiento, con los mismos indicadores según el tipo de población de estudiantes y el tipo de establecimientos de que se trate? ¿sería posible hacerlo según tipos culturales juveniles?

Indagar a través del discurso de los jóvenes escolares permitiría a los profesionales problematizar las propias nociones de cambio social, desigualdad e injusticia. Para los gestores de políticas públicas plantea el desafío de escuchar las demandas de los jóvenes escolares de enseñanza media.

\section{Objetivos proyecto general}

a) La investigación propone comprender, desde la perspectiva de los sujetos de estudio y sus representaciones

Comprender el significado que tiene la escuela para los jóvenes escolares chilenos, como posible espacio favorecedor del sentimiento de inclusión o exclusión social.

Proporcionar elementos para la construcción de propuestas de políticas públicas y programas para la juventud escolar chilena en función de apoyar una educación con sentido de calidad y equidad para los jóvenes escolares.

En el sentido de generar preguntas que trasciendan la investigación y de alguna manera guiarían los tópicos o los ámbitos a discutir con los jóvenes escolares en los focus group de la investigación global.

- ¿cuánto participa en la escuela? ¿en qué? ¿para qué? ¿con qué sentido? ¿con qué grado de satisfacción personal? 
- ¿la escuela lo insta a participar o es iniciativa personal?

- ¿qué tanto la escuela está influyendo en las trayectorias de vida de estos jóvenes?

- ¿cómo se inscriben los procesos sociales en las existencias individuales?

- De aquí entonces ¿cómo se inscriben los procesos sociales en la trayectoria escolar cuando ésta se encuentra marcada por la exclusión social?

- ¿qué significa ser joven en esta sociedad? ¿sientes que formas parte de la sociedad chilena actual? ¿en qué sentido? ¿qué indica que eres parte, qué indica que no eres parte?

- ¿cuándo te sientes integrado en un espacio social y cuándo no? ¿en relación a qué?

Es reconocido, cada vez con mayor fuerza el hecho que los jóvenes que cursan enseñanza media, requieren de una preocupación por parte de los profesionales de la educación, que se acerquen a su mundo, a los significados de sus experiencias y por sobre todo al significado de la experiencia escolar, la forma que construyen y construyen su experiencia, la forma en que construyen su cultura juvenil y proyectan los cambios de la cultura general en la que estamos todos insertos. Acercarnos a la comprensión profunda de la experiencia subjetiva de los escolares jóvenes, de su experiencia escolar, permitiría promover intervenciones y políticas sustentables, en planos tan polémicos como lo es el de las valorizaciones, las que se entenderán como orientaciones de acción consensuadas. Hoy en día, en la realidad de los establecimientos educacionales se convive en un mismo espacio, mundos lejanos, un espacio de interacciones cargadas de asimetría, donde se generan recurrentes incomprensiones y conflictos, tanto explícitos como implícitos.

En este espacio, donde conviven diferentes culturas, éstas son construidas,

No solamente a partir de los procesos internos, sino que también, sobre la base de los significados y creencias que se encuentran en la sociedad en que ésta se encuentra inserta. De este modo, esta construcción cultural se encuentra afectada por el cambio cultural y social que enfrentamos como sociedad (Ríos, 2004:8).

En este sentido, basándose en el objetivo del pretendido estudio, el enfoque y paradigma que acompañaría la investigación resulta cohe- 
rente y adecuado, el cualitativo. Sin embargo, existen estudios de carácter cuantitativo como los de Oscar Dávila, que deberían complementar éste en su discusión y conclusiones.

Por otra parte, en términos del sujeto de estudio, continúa siendo el joven en edad escolar. En función de considerar la exclusión social como una problemática del sistema social más amplio, un problema cultural, no necesariamente serán jóvenes de niveles socioeconómicos bajos, sino que distintos tipos de jóvenes dentro de la diversidad cultural juvenil. Sin embargo, en este punto es importante preguntarse si los casos son sólo los estudiantes o las escuelas; desde la definición planteada, sería el joven escolar en relación a la escuela. Debería existir un cruce entre jóvenes y dependencias diferentes de establecimientos.

Finalizando, podría decir que la realización de un estudio como éste, aportaría teóricamente a la reflexión que constantemente se realiza en función de las políticas públicas en cuanto a los jóvenes y al sentido de la escuela para ellos(as); así mismo, constituiría un aporte en la medida que entregara información desde el ámbito subjetivo en la construcción de la percepción de inclusión o exclusión social, considerando toda las precisiones realizadas.

Pensando en una posible respuesta a la pregunta central realizada para la investigación, podría suponer que en un primer plano, casi desde el sentido común, aparecería la problemática de la segmentación social reflejada en la segmentación escolar. Sin embargo, la tendencia a partir de esta primera reflexión, es que la respuesta de los jóvenes o la percepción de los jóvenes, implicaría inevitablemente el cuestionamiento al sentido de la escuela, aparecería tal vez la crítica respecto de que la escuela se ha sometido a las reglas del mercado, y como tal, sería difícil desvincularla a los criterios en base a los cuales se mueve el mercado, en este sentido, la variable nivel socioeconómico (NSE) y trabajo serían centrales.

En este mismo plano, podría pensar que la escuela aparecería altamente instrumentalizada para los jóvenes, es decir, «sirve» en la medida que le pueda dar continuidad a un proyecto económico, a una medida de subsistencia, no necesariamente a un proyecto de vida, como el que propone la educación chilena. Dicho escenario podría significar hacer caer todo aquel proyecto que pretende el logro de objetivos transversales comunes ¿cuántos sueños vende la escuela, cuántos de estos sueños pueden ser alcanzados por la mayoría de los jóvenes escolares? ¿cuántos de estos sueños son de interés de los jóvenes? Un joven de clase alta por ejemplo, podría perfectamente per- 
cibir que la educación chilena no cumple con sus expectativas de vida, con sus expectativas de sociedad y por lo tanto buscaría las formas de vincularse y acercarse a espacios donde respondan a estas expectativas, sean nacionales o internacionales.

La gran mayoría de los jóvenes participan de la escuela, lo que hace descartar aquello como variable a considerar para verificar inclusión o exclusión, quedaría por preguntar en qué sentido la escuela es portadora, generadora o inspiradora de la percepción de inclusión y/o exclusión social para los jóvenes, restando a ello, ya el hecho de ser jóvenes, razón que les imprime a estos sentimientos de exclusión y discriminación.

\section{SANTIAGO (CHILE), JUNIO 2008}

RECIBIDO: JULIO 2008

ACEPTADO: SEPTIEMBRE 2008

\section{REFERENCIAS BIBLIOGRÁFICAS}

ANTIVILO, CAVIERES y VALDIVIESO (2006): «La exclusión social: un estudio psicosocial sobre las percepciones juveniles».

En: www.uisek.cl.

ASSAÉL, J. y A. M. CERDA (1998): «Contenidos valóricos y normativas que construyen los jóvenes en el espacio escolar: un estudio etnográfico» (Proyecto Fondecyt $\mathrm{N}^{\circ}$ 971181). Santiago: Programa Interdisciplinario de Investigación en Educación.

CARrasco, J. y M. GonZÁles (S/F): «Entrevista a Gabriel Salazar». Revista Talión, 4, (6).

DÁvila, GHiardo y Medrano (2005): Los desheredados. Trayectorias de vida y nuevas condiciones juveniles. Valparaíso: Ediciones CIDPA.

DuBET, F. (2002): Le déclin de l'institution. Paris: Editions du Seuil.

GHIARDO y DÁVILA (2007): «Elementos para reconstruir trayectorias laborales juveniles desde las encuestas nacionales de juventud». Revista Observatorio de Juventud $\mathrm{N}^{\circ} 15$. Santiago: INJUV.

IMAZ, J. E. (2002): «La educación del futuro y los valores». Ponencia impartida en el ciclo «Debates en educación», organizado por la Fundación Jaume Bofill y la UOC. En: http://contexto-educativo.com.ar.

INJUV (2000): La eventualidad de la inclusión. Jóvenes chilenos al comienzo del nuevo siglo. Tercera encuesta nacional de juventud. SantiaGO: INJUV. 
MolinA (2003): «Calidad y valores». En HEviA (editor): La educación en Chile, hoy. Santiago: Universidad Diego Portales.

REDONDO, (2000): «La condición juvenil: entre la educación y el empleo». Última Década $\mathrm{N}^{\circ} 12$. Viña del Mar: Ediciones CIDPA.

Rios, T. (2004): «La configuración de sentidos en un grupo de jóvenes liceanos de sectores populares: un acercamiento hermenéutico al mundo juvenil escolar». Tesis doctoral no publicada. Santiago: Pontificia Universidad Católica de Chile.

RoJAS, M. T. (2004): «Equidad escolar en Chile. Un estudio de casos sobre las racionalidades que poseen diferentes actores educativos acerca de la equidad escolar». Tesis doctoral no publicada. Santiago: Pontificia Universidad Católica de Chile y a L'Ecole Doctorale de la Faculte des Sciences Humaineset Sociales de L'Universite Rene Descartes Paris 5, Sorbonne.

ROJAS, X. (2006): «El proceso de socialización en valorizaciones de alumnos y alumnas de enseñanza media en Chile: una aproximación desde el análisis de la experiencia escolar». Tesis doctoral no publicada. Santiago: Pontificia Universidad Católica de Chile.

ZARZURI, R. y R. GANTER (2005): Jóvenes: la diferencia como consigna. Santiago: Ediciones CESC.

— - y (2002): Culturas juveniles, narrativas minoritarias y estética del descontento. Santiago: Ediciones UCSH. 